\title{
Acti Ball+: an Akkermansia muciniphila useful tool and innovative approach to lose weight
}

\begin{abstract}
Introduction: The dominant treatments for obese patients are the anti-obesity drugs. We propose a mixture of vegetable fibers and fructo-oligosaccharides as a valid alternative with limited side effects.

Methods: An evaluation of ActiBall's action on fats and carbohydrates was performed in vitro assay using pancreatic enzymes. The effect of the 2-fiber-Mix on each of the various bacterial groups present in the human gut was expressed as the ratio of bacterial abundance after $48 \mathrm{~h}$ versus the start of the incubation. The influence on the microbiota was evaluated using the Simulator-of-the-Human-Intestinal-Microbial-Ecosystem (SHIME®).

Results: The fibers mixture display a higher capacity than control to trap fats contained in the tested food items: for chocolate $(+24 \%)$, chips $(+76 \%)$ and pistachios $(+32 \%)$, decreasing the kcal by half. The mixed fibers resulted in a Firmicutes over Bacteroidetes ratio that is rather associated with a lean versus an obese gut microbiome. The results of the SHIME $®$ assays have shown that it regenerates the populations of $A$. muciniphila.

Conclusion: The fiber complex acts upstream as calorie storage thus reducing assimilation of fats and sugars present in meals. The same fiber complex stimulates microflora growth and restores beneficial intestinal microflora, resulting in typical prebiotic effects (decreased $\mathrm{pH}$, increased production health promoting SCFA, increased health gut microflora).
\end{abstract}

Keywords: vegetable fibers, medical treatment, fats, carbohydrates, microbiota, prebiotic effect
Volume II Issue 4 - 2020

\author{
Christophe Bastid,' Marius Nedelcu, ${ }^{1,2}$ \\ Thierry Manos,' Patrick Noel,' Anamaria \\ Nedelcu, ${ }^{2}$ Laurent Didden' \\ 'Bouchard Elsan Clinic, France \\ ${ }^{2}$ Elsan Clinic Saint Michel, France
}

Correspondence: Laurent Didden, Clinique Bouchard, 77 rue du Docteur Escat 13006 Marseille, France, Tel 0033695950965 , Email didden.laurent@neuf.fr

Received: July II, 2020 | Published: August 31, 2020

\section{Introduction}

The Medical approach for the treatment of morbid obesity disease has shown its limitations compared to the surgical treatment. ${ }^{1-3}$ Still, as this dreaded disease is multifactorial, the medical treatment will continue to have a place for non-surgical patients, as adjuvant for new endoluminal approach, or for patients with minimal weight recidivism. There are many dietary supplements on the market containing plants, vitamins and minerals or other substances. These supplements are intended to help improve consumers' nutritional intake in order, for example, to help them lose weight, improve digestion, embellish hair. However, there is a limited choice of effective and safe agents for reducing dietary fat consumption. Laboratoires ClémaScience has developed ACTI BALL+ ${ }^{\circledR}$, an oral medical device composed of a patented natural fiber complex which constitutes an innovative solution for overweight and obese people in the management of their weight. Its main benefic actions are the trapping of fats, sugars and so calories, the regulation of satiety and anti-snacking effect. It has also demonstrated prebiotic properties with regards to stimulating growth of beneficial intestinal microflora, thus enhancing ACTI BALL+®'s action on weight-loss and calorie-reduction. Dietary fibers present at least one of the following properties: an increase in stools production, the reduction of pre-prandial cholesterol levels and the reduction of post-prandial blood glucose and/or insulin levels, and stimulation of colonic fermentation. ${ }^{4}$

The purpose of the current manuscript is to summarize the existing data regarding the new product and to identify potential indications for patients with obesity disease. ACTI BALL $+\circledR$ has been evaluated to demonstrate its useful approach in weight loss and prevention of obesity in overweight people.

\section{Methods}

The following studies have been conducted in order to validate the actions and mechanisms claimed by ACTI BALL $+($ complex with regards to anti-fat action, anti-sugar action, anti-calorie action, antihunger action (satiety and anti-snacking), and prebiotic action.

\section{Evaluation of the effectiveness in capturing dietary fats}

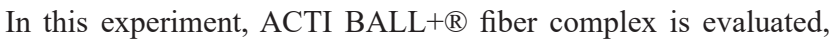
and the fatty products tested are chocolate, pistachios, brioches and chips. A control assay was performed using an equivalent quantity of a commercially available product Calorilight ${ }^{\circledR}$. All fatty products are prepared in a similar manner: fatty products + water $(1: 1 \mathrm{w} / \mathrm{v}$ for chocolate; $1: 10 \mathrm{w} / \mathrm{v}$ for brioche and 1:6 w/v for chips and pistachios) are heated, homogenized, heated again and filtered. Lipidic supernatant is pipetted in enough quantities to constitute the basic fatty component of each assay.

\section{Evaluation of ACTI BALL+@'s action on fats and carbohydrates}

An in vitro assay was designed using pancreatic enzymes and in conditions of $\mathrm{pH}$ and temperature close to those of the digestive tract. The products under study are incubated with enzymes (amylase, lipase from pork pancreatin) in a buffer solution at $\mathrm{pH} 6.5$ and $37^{\circ} \mathrm{C}$. The lipase initial activity is $2000 \mathrm{U}$ tributyrin/ml $(250 \mu \mathrm{g}$ lipase/ $\mathrm{mL}$ ). This activity corresponds to the average activity measured at the intestinal level during a meal and is used as reference for the pancreatin concentration to implement during the test. 


\section{Evaluation of anti-caloric action of ACTI BALL+®}

This evaluation was performed using the same in vitro assay as above (that mimics conditions in the digestive track). The lipids present in this test meal are mainly composed of Triglycerides (TG). Considering a mean molar mass of $885.4 \mathrm{~g} / \mathrm{mol}$ for the TG contained in the meal, we thus obtain an initial concentration of TG of 33.88 $\mathrm{mM}$.

\section{Evaluation of the anti-snacking effect of ACTI BALL+@}

This evaluation was performed using the same in vitro assay as above (that mimics conditions in the digestive track), the same methods (cf 3.2 Material \& Methods), and the test meal was also Fortimel Energy.

Evaluation of the effect of two ffibers blends in short and long term experiments using the Simulator of the Human Intestinal Microbial Ecosystem (SHIME $\AA$ ). The following short-term batch experiments were designed to assess how the fiber properties of ACTI BALL $+\mathbb{R}$ complex influence the microbiota in terms of microbial fermentation, activity and composition using the SHIME ${ }^{\circledR}$ system. At the start of the short-term in vitro colonic incubation, the ACTI BALL+ ${ }^{\circledR}$ complex was added to sugar-depleted SHIME nutritional medium containing basal nutrients that are present in the colon together with a freshly prepared human faecal inoculum of the selected donor. Incubations were performed anaerobically at $37^{\circ} \mathrm{C}$ during $48 \mathrm{~h}$ and under continuous stirring $(90 \mathrm{rpm})$. This allowed assessing the specific effect of the complex on overall microbial fermentation, specific microbial metabolites and microbial community composition. In order to account for biological variability, all tests were performed in triplicate, resulting in 9 independent incubations.

The SHIME long-term experiment for this study consisted of 3 stages: during the 2-week stabilization period, the colon reactors are inoculated with fresh faecal sample from donor (obese, low in Akkermansia muciniphila levels) identified during pre-screening. Thus, the microbial community can differentiate in the different reactors depending on local environmental conditions. Basic nutritional matrix was provided to the SHIME to support the maximum diversity of the gut microbiota present in the faecal inoculum. A 2-week reference period follows, during which the standard SHIME nutrient matrix was further dosed for a period of 14days. Analysis of samples in this period allows determining the baseline microbial community composition and activity in the different reactors, which will be used as a reference to compare the results from the probiotic treatments. During the 3-week treatment period, the SHIME reactor was operated under nominal conditions, but with a diet supplemented with the prebiotic product (ACTI BALL $+\AA$ ) at final target dose of $2.4 \mathrm{~g} / \mathrm{L}$ while in vitro fermentations.

Microbial community activity is evaluated 3x/week (during 2 control and 2 treatment weeks) by measuring SCFA (concentration of acetic acid, propionic acid and butyric acid analyzed) and lactate analysis (lactate is produced by lactic acid bacteria and decreases the $\mathrm{pH}$ of the environment). Microbial community composition is evaluated $1 \mathrm{x} /$ week by quantifying the following groups in the lumen and mucus via qPCR: Akkermansia muciniphila, Bacteroidetes, Firmicutes, Lactobacillus spp, Bifidobacterium spp.

The final target dose was 6 capsules/day resulting in the intake of $2 \mathrm{~g} /$ day, corresponding to an equivalent dose of $2.4 \mathrm{~g} / \mathrm{L}$ while in vitro fermentations.

\section{Results}

The results of the mixture's capacity to capture fat mass compared to the control product are summarized in Table 1, which represents mean values of the results obtained comparing ACTI BALL $+(\mathbb{R}$ and Calorilight ${ }^{\circledR}$ with regards to their capacity to trap fats. In all but one case, ACTI BALL $+\circledR$ complex displays a higher capacity than Calorilight ${ }^{\circledR}$ to trap fats contained in the food items that have been tested. This test has allowed us to confirm the high fat capturing strength contained in ACTI BALL $+{ }^{\circledR}$ fiber mixture. This is particularly high $(\mathrm{Cg}>10)$ for chocolate, chips and pistachios, and it leads us to believe that the capturing capacity of this mixture is greater on vegetal fats, because the result on brioche is less important.

Table I Results from in vitro assay comparing the capture of dietary fats in various items by ACTI BALL® vs Calorilight ${ }^{\circ}$ (mean values)

\begin{tabular}{|c|c|c|c|c|c|c|c|}
\hline Fatty food & $\begin{array}{l}\text { Fiber } \\
\text { type }\end{array}$ & $\begin{array}{l}\text { Mean quantity } \\
\text { of lipids }\end{array}$ & $\begin{array}{l}\text { Quantity of } \\
\text { mixture total/fibers }\end{array}$ & $\begin{array}{l}\text { Mean quantity } \\
\text { Supernatant }\end{array}$ & $\begin{array}{l}\text { Mean } \\
\text { Cg }\end{array}$ & $\begin{array}{l}\text { Standard } \\
\text { deviation }\end{array}$ & $\begin{array}{l}\text { Acriball vs. } \\
\text { Calorilight }\end{array}$ \\
\hline \multirow{2}{*}{ Chocolate } & Control & 3104.6 & $270.2 / \mathrm{na}$ & 673.5 & 8.99 & 0.219 & \multirow{2}{*}{$+24 \%$} \\
\hline & ActiBall & 3044.8 & $266.2 / 191.4$ & 78.1 & 11.14 & 0.128 & \\
\hline \multirow{2}{*}{ Brioche } & Control & 3058.0 & 269.9/na & 176.2 & 10.68 & 0.093 & \multirow{2}{*}{$-38 \%$} \\
\hline & ActiBall & 3033.8 & $266.2 / 191.3$ & 979.4 & 7.72 & 0.584 & \\
\hline \multirow{2}{*}{ Chips } & Control & 2997.1 & $276.1 / \mathrm{na}$ & | 427.8 & 5.684 & 0.132 & \multirow{2}{*}{$+76 \%$} \\
\hline & ActiBall & 2955.3 & $263.2 / 189.3$ & 316.8 & 10.01 & 0.192 & \\
\hline \multirow{2}{*}{ Pistachios } & Control & 3050.3 & $270.6 / \mathrm{na}$ & 759.7 & 8.463 & 0.151 & \multirow{2}{*}{$+32 \%$} \\
\hline & ActiBall & 2999.7 & $262.8 / 188.7$ & 71.46 & 11.14 & 0.38 & \\
\hline
\end{tabular}

In The second test was performed in order to evaluate the capacity of ACTI BALL $+\circledR$ to trap sugars as well as fats in an in vitro assay. The test meal used was Fortimel Energy. At the end of 4 hours, the impact of the various combinations on lipolysis at the end of digestion is a $13,2 \%$ inhibition with ACTI BALL $+\circledR$ complex. The impact of ACTI BALL+® complex on sugar hydrolysis is $20.3 \%$ and it is summarized in Table 2. 
Table 2 Effect of ACTI BALL+ on hydrolysed sugar content vs control group

\begin{tabular}{lll}
\hline Time $(\mathrm{min})$ & Control group $(\mathrm{mg} / \mathrm{ml})$ & ACTIBALL $(\mathrm{mg} / \mathrm{ml})$ \\
\hline $0 \mathrm{~min}$ & 43.59 & 43.59 \\
$15 \mathrm{~min}$ & 50.11 & 42.22 \\
$60 \mathrm{~min}$ & 76.20 & 48.74 \\
$240 \mathrm{~min}$ & 75.85 & 60.41
\end{tabular}

At the end of 4 hours, $147 \mathrm{kcal}$ (lipids and carbohydrates) remain in the medium in the presence of ACTI BALL+ ${ }$, to which are added calories ingested from proteins $(72 \mathrm{kcal})$. Since these calories have not been measured, we conclude that it remains at $100 \%$ in the final medium. Thus, at the end of four 4 hours, a total of $21 \mathrm{kcal}$ remain in the medium. Of the $450 \mathrm{kcal}$ initially ingested by the test meal, ACTI BALL $+{ }^{\circledR}$ would capture $231 \mathrm{kcal}$, or half of the total caloric count. We can conclude that for the daily equivalent of two test meals, ACTI BALL $+\circledR$ captures a total of $462 \mathrm{kcal}$ per day. By progressively slowing the hydrolysis of complex sugars during meals, ACTI BALL+ $($ allows for a continuous intake of reducing sugars in the body, thereby promoting the feeling of satiety. Hunger and food cravings would then be reduced.

The consumption of acid and base reflects the overall microbial activity. Indeed, bacteria may produce increased amount of short-chain fatty acids (SCFA) and lactate. As a consequence, the environment will acidify. ACTI BALL $+\AA$ was fermented, as followed from the increased base consumption in ascending, transverse and descending colon (AC, TC and DC) during the treatment (Figure 1). When averaged over the control and treatment period, these increases were significant in the AC and TC. The higher base consumption of ACTI BALL $+\circledR$ in the DC indicates that a proportion of the fiber complex resisted fermentation in the $\mathrm{AC}$ and therefore also reached the distal colon regions.

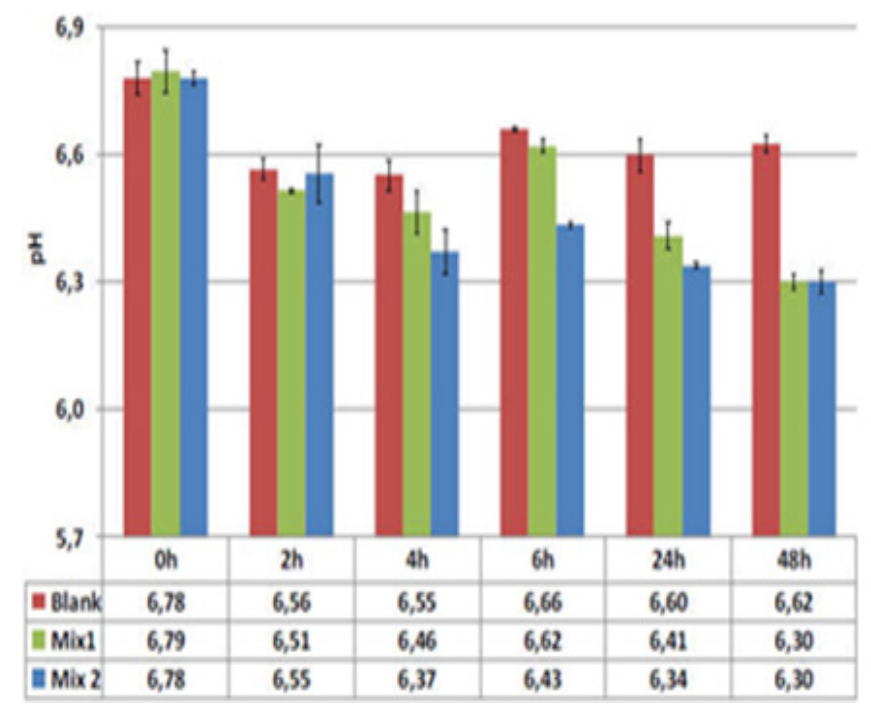

Figure I Average base-acid consumption over the entire control $(n=6)$ and treatment period $(n=6)$. Results are provided for the ascending colon $(A C)$, transverse colon (TC) and descending colon (DC).

Gas production is too a major endpoint of fermentative activity by gut bacteria. In this study, gas production was the strongest during the first $24 \mathrm{~h}$ of incubation (Figure 2).

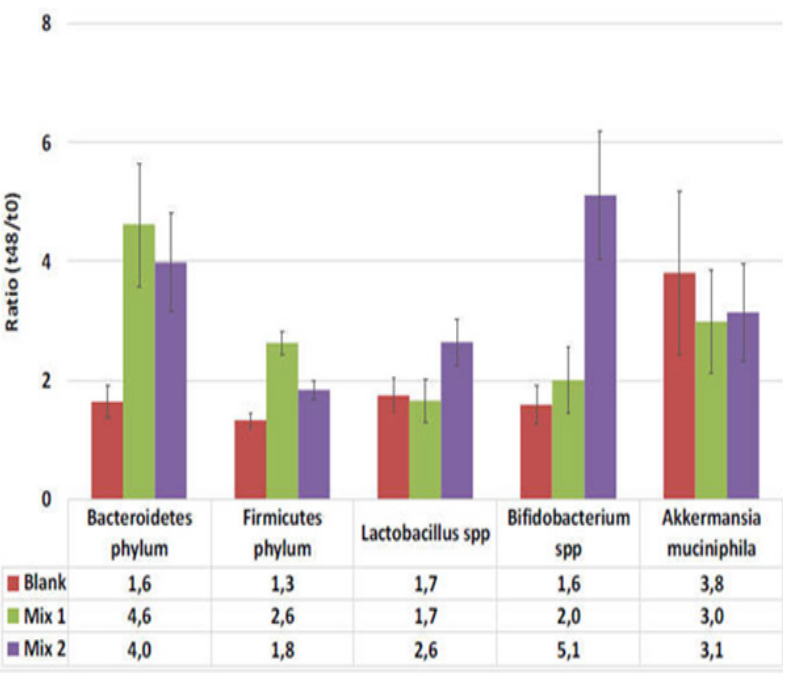

Figure 2 Average gas production $(\mathrm{kPa})( \pm \mathrm{STDEV})$ during a control incubation and upon dosing Acti Ball+ to the ascending colon microbiota $(n=3)$ during the control period.

Regarding the long-term effect, during the control period, SCFA levels were very stable (on average $91 \%$ similar between consecutive time points in control period), clearly indicating stability of the microbial community in terms of activity and composition. The treatment with ACTI BALL $+R$ was associated with a gradual increase in total SCFA production in the ascending, transverse and descending colon. This increase was mainly due to an increased production of acetate and propionate (Figure 3).

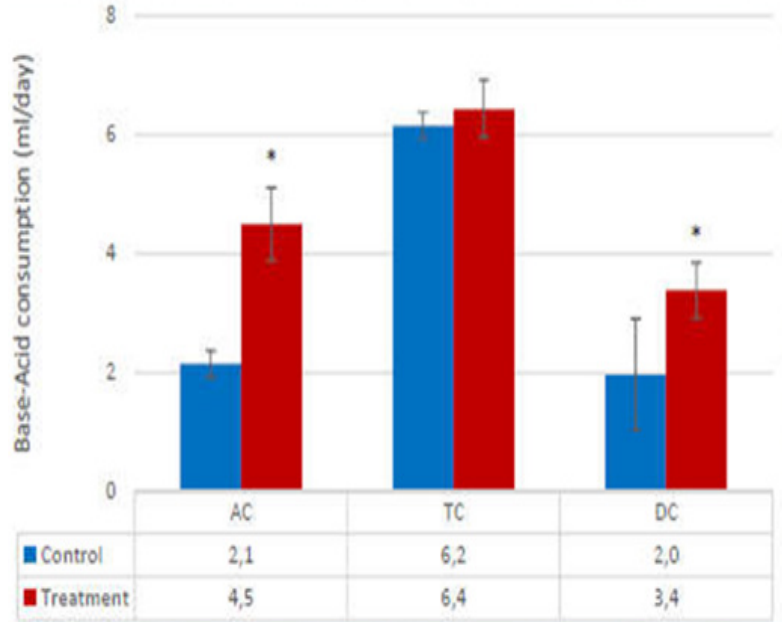

Figure 3 Effect of Acti Ball+ on acetate production in the ascending (AC), transverse (TC) and descending colon (DC). Results are given as the average acetate production over the control $(n=6)$ and treatment $(n=6)$ period (*indicates statistically significant differences relative to the preceding period).

The effects of ACTI BALL+ ${ }^{\circledR}$ on each of the various bacterial groups present in the human gut are expressed as the ratio of bacterial abundance after $48 \mathrm{~h}$ versus the start of the incubation. The results illustrate that ACTI BALL+® favored growth of Bacteroidetes in the three colon regions. Since this phylum contains the most abundant propionate producers, stimulation of Bacteroidetes in the three colon regions can be related to the increase in propionate concentrations observed earlier. However, the levels of Firmicutes have no registered 
significant changes. The decrease in the ratio of Firmicutes to Bacteroidetes was statistically significant in the $\mathrm{AC}$ and $\mathrm{DC}$ regions (Figure 4). Furthermore, ACTI BALL $+\AA$ had a strong stimulatory effect towards on Lactobacilli and especially Bifidobacteria (Figure 5). Lactobacilli and Bifidobacteria are regarded as beneficial saccharolytic bacteria. Both groups are capable of producing high concentrations of lactate.

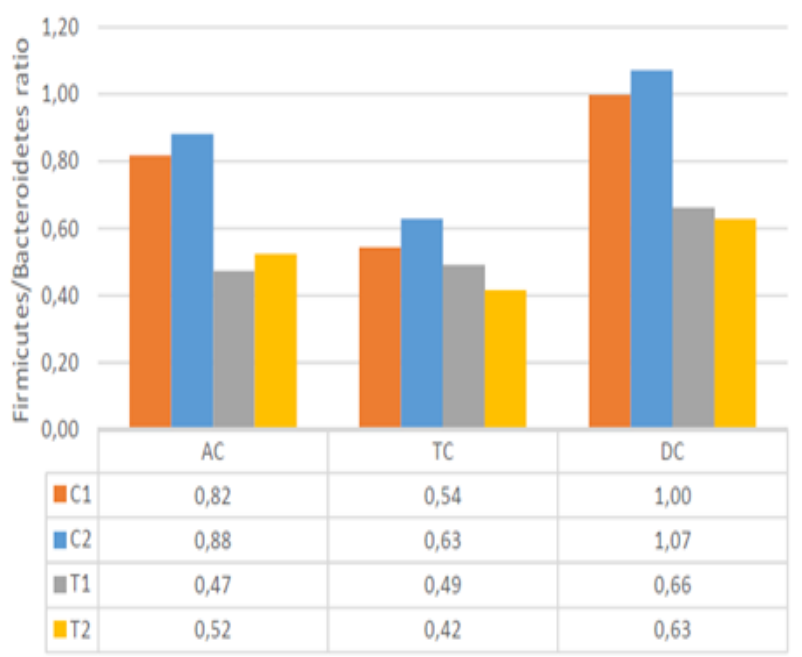

Figure 4 Firmicutes/Bacteroidetes ratio during the control $(\mathrm{Cl}-2=$ contro weeks I-2) and treatment (TI-2= treatment weeks I-2) periods in the three regions of the colon.

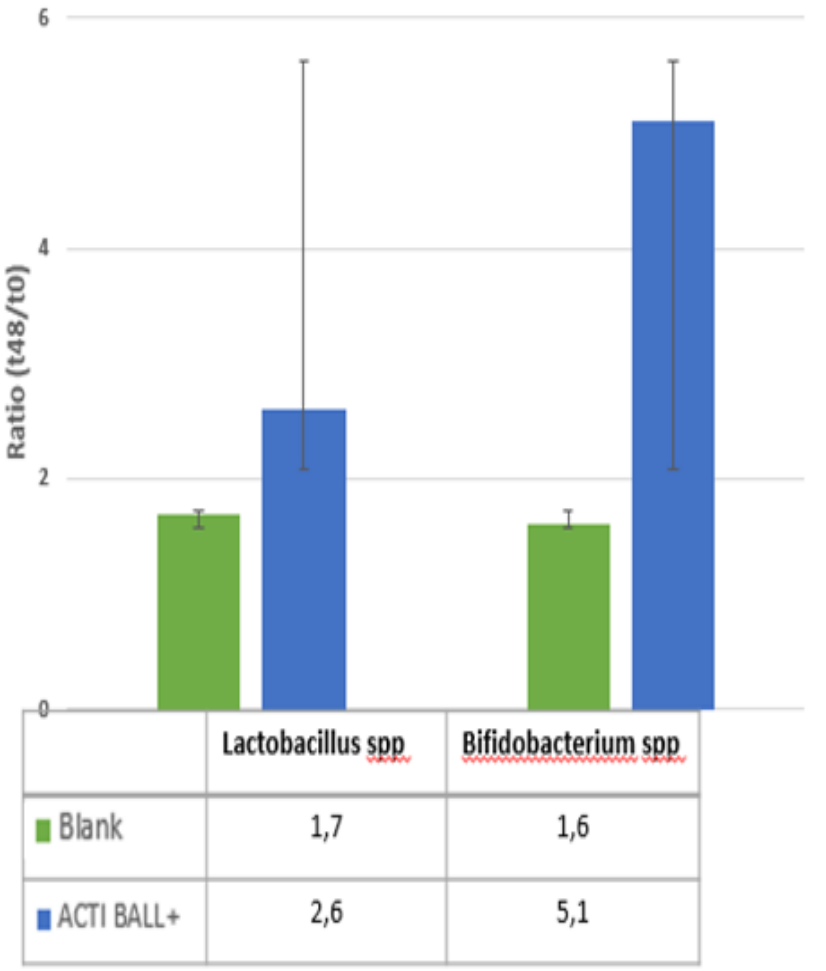

Figure $\mathbf{5}$ The effect of the fiber mixtures and the blank background medium on growth of Lactobacilli and Bifidobacteria. The effect is expressed as the ratio of bacterial abundance after $48 \mathrm{~h}$ versus the start of the incubation.

During the prescreening experiment, the donor selected had low in Akkermansia muciniphila levels compared to other bacterial populations. In contrast to the control period, where Akkermansia muciniphila was only detected in the TC, this group was detected in all colon reactors during the treatment period. These results suggest a possible enrichment of Akkermansia muciniphila in the lumen upon treatment with ACTI BALL+ ${ }^{\circledR}$ (Figure 6).

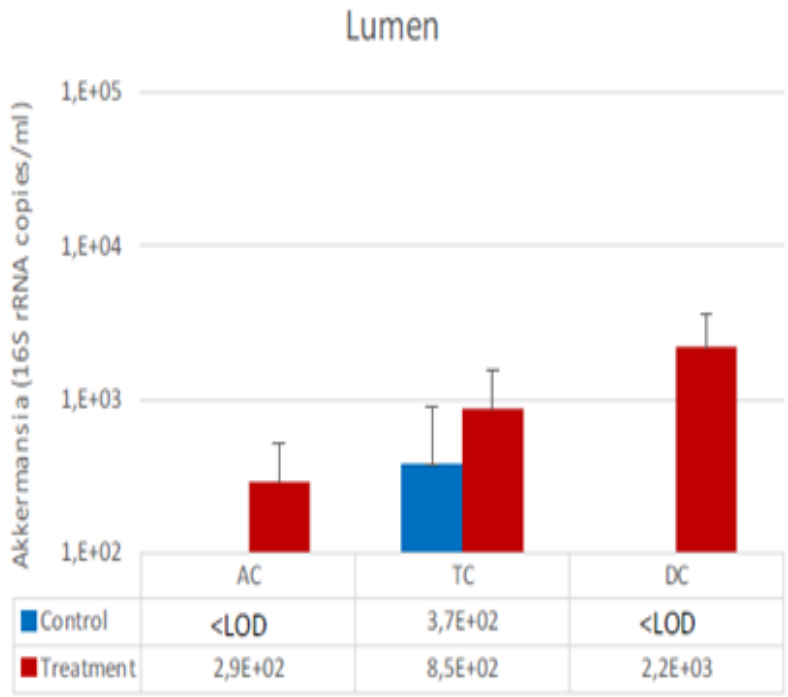

Figure 6 Effect of Acti Ball+ on luminal and mucosal Akkermansia muciniphila levels (I6S rDNA copies/mL) in the ascending (AC), transverse (TC) and descending colon (DC) reactors. Represented values are averaged over the entire control and treatment period.

\section{Discussions}

Obesity disease is undoubtedly multifactorial in origin, and acquired influences (urbanization of the world's population, increasingly sedentary lifestyle, and a nutritional transition to processed foods) probably exceed genetic factors in its causation. Even if it is complex, obesity remains largely a preventable disease, ${ }^{5}$ affecting, along with overweight, over a third of the world's population today. ${ }^{6,7}$ If secular trends continue, by 2030 an estimated $38 \%$ of the world's adult population will be overweight and another $20 \%$ will be obese. ${ }^{8}$

Of course, lifestyle modifications represent the cornerstone of preventing morbid obesity disease and the overweight, but the same lifestyle modifications also represent an adjunct to bariatric surgery. Endoscopic bariatric therapy may be a useful alternative, and it provides greater efficacy with lower risks than conventional surgical procedures. It includes intragastric balloons, gastroplasty techniques, aspiration therapy, and gastrointestinal bypass sleeves. The singular and separate approach of lifestyle modifications is prone to fail, and it should not be encouraged as an isolated treatment for morbidly obese patients. The other major limitation is that it is time consuming and imposes an economic burden on for patients and physicians. Considering the current rates of worldwide obesity, this separate approach is extremely limited and highly ineffective, weight regain being very common once the patient has completed a lifestyle intervention program. ${ }^{9-11}$ In a similar manner, to prove its efficiency, pharmacotherapy should be used as an additional tool for both lifestyle modifications and bariatric surgery. ${ }^{12}$ As obesity disease is a multifactorial disease, the treatment should include the concept of complementarity between these methods of treatment than to present them as separate options.

Although anti-obesity drugs are the dominant treatment used by obese patients, they are limited by serious side effects such as 
negative mood changes, possible liver damage, gastrointestinal or cardiovascular complications, and the potential for drug abuse and dependency for some people. These issues have resulted in a bottleneck in developing a safe and effective weight control strategy. Consequently, ACTI BALL $+\circledR$, composed of a mixture of vegetable fibers and fructo-oligosaccharides, is proposed as a valid alternative with limited side effects. Previous reviews have shown the health benefits of dietary fiber, as higher intakes of these fibers are linked to less cardiovascular disease, and that fibers also play a role in gut health by helping to maintain regularity of colonic functioning. For decades, studies to discover the potential of plants and their phytochemicals in controlling obesity have been conducted. These focused on several promising modes of actions ${ }^{13,14}$ such as inhibition of lipase activity, ${ }^{15}$ appetite suppression/satiety induction, ${ }^{16}$ thermogenesis, regulation of lipid metabolism, and inhibition of adipogenesis. ${ }^{17}$ The results from these various in vitro experiments have shown that ACTI BALL $+\AA$ fiber complex reduces sugar absorption and thus caloric intake by $20 \%$ (462kcal captured per day), and that this fiber mixture also has a high capacity to capture fats issued from foods like chocolate, chips, in laboratory conditions. It is postulated that dietary fat bound to a fiber complex is not available for digestion and is eventually eliminated as it is, thus helping to reduce energy intake and promote weight loss.

The concept of prebiotic is based on the possibility of favorably modulating the gut microbiota composition to maintain and/or promote health, using nutrients such as nondigestible, fermentable carbohydrates. ${ }^{18}$ Prebiotics are defined as «selectively fermented ingredients that allow specific changes in the gastrointestinal microflora that confers benefits to the host's health and well-being». ${ }^{19}$ Non-digestible oligosaccharides (NDO) thus resist digestion and absorption in the human small intestine so that they are completely or partially fermented in the large intestine. These prebiotics beneficially affect the host by selectively stimulating the growth and/or activity of beneficial bacteria in the colon, thereby improving host health.

According to the literature,,$^{20,21}$ we decided to use SHIME®, as a system for measuring these parameters which provides the possibility to work with a stabilized microbiota community and to regularly collect samples from the different intestinal regions for further analysis. By measuring the acid/base consumption throughout the experiment, the potential fermentation of the prebiotic products can be estimated. Since gasses are a major endpoint of fermentative activity by gut microbes, changes in gas production provide an indication of the overall fermentation profile. SCFA production results from carbohydrate metabolism in the colon and is related with various health effects; and usually the most abundant SCFAs are acetate, propionate and butyrate.

According to Kallus \& Brandt ${ }^{22}$ in a murine model, the majority of the intestinal microbiome consists of two bacterial phyla, the Bacteroidetes and the Firmicutes, of which the relative abundance differs among lean and obese mice. The obese mouse seemed to have higher proportion of Firmicutes to Bacteroidetes than the lean mouse. Similar findings were observed in obese humans compared to lean subjects. Thus, the ratio Firmicutes/Bacteroidetes could be considered as an indicator linked to obesity. With respect to the current assay, the treatment with ACTI BALL+ $₫$ thus resulted in a Firmicutes over Bacteroidetes ratio that is rather associated with a lean versus an obese gut microbiome.

But the most attractive species is Akkermansia muciniphila, a mucin-degrading agent which exhibits gut beneficial properties and that is found in lower concentrations in obese individuals. Initially described in $2005,{ }^{23}$ it has become very popular since the discovery of its probiotic properties. Moreover, it was shown that there was a strict correlation between gut concentrations of this bacterium and gut inflammation and permeability; and abundance of A. muciniphila was associated with decreased metabolic endotoxaemia and adipose tissue inflammation due to the restoration of the original mucus layer and specific antimicrobial peptides..$^{24}$ Zhou et al. ${ }^{25}$ have showed that in vitro culture experiments, showed that prebiotics and dietary fibers as FOS significantly promoted $A$. muciniphila's growth. The results of the SHIME $®$ assays have shown that ACTI BALL $+\circledR$ fiber complex regenerates concentrations of $A$. muciniphila, and can thus be used as a factor participating in the management of weight and other pathologies.

\section{Conclusions}

The fiber complex acts upstream as calorie storage, thus reducing assimilation of fats and sugars present in meals. Its unique fiber complex swells in contact with the food bolus and forms a viscous gel in contact with water that coats the intestine walls. The micropores that are present alongside the insoluble fibers retain water, dietary fats and sugars by capillarity. It's their high capacity to swell and retain water and fats along their transit throughout the stomach and intestine promotes satiety and thus reduction of food intake, natural elimination of fats in stools and better transit. All these properties act in combination to limit calorie intake from food and contribute to weight loss. The same fiber complex stimulates microflora growth and restores beneficial intestinal microflora, resulting in typical prebiotic effects (decreased $\mathrm{pH}$, increased production health promoting SCFA, increased health gut microflora). Additional research is needed to

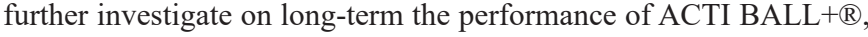
as well as its long-term beneficial effect on stimulating the growth of health-promoting microflora such as $A$. muciniphila.

\section{Statement of ethics}

All procedures performed in studies involving human participants were in accordance with the ethical standards of the institutional and/ or national research committee and with the 1964 Helsinki declaration and its later amendments or comparable ethical standards. For this type of study formal consent is not required.

\section{Acknowledgments}

None.

\section{Conflicts of interest}

The authors have no conflicts of interest to declare.

\section{Funding sources}

No financial and material support for this research and work were used.

\section{Author contributions}

The contributions of each author included

1. Substantial contributions to the conception or design of the work; or the acquisition, analysis, or interpretation of data for the work: Christophe Bastid, Thierry Manos, Laurent Didden.

2. Drafting the work or revising it critically for important intellectual content: Christophe Bastid, Marius Nedelcu, Anamaria Nedelcu. 
3. Final approval of the version to be published: Christophe Bastid, Marius Nedelcu, Thierry Manos, Anamaria Nedelcu, Laurent Didden.

4. Agreement to be accountable for all aspects of the work in ensuring that questions related to the accuracy or integrity of any part of the work are appropriately investigated and resolved: Christophe Bastid, Thierry Manos, Marius Nedelcu.

\section{References}

1. Courcoulas AP, Gallagher JW, Neiberg RH, et al. Bariatric Surgery vs. Lifestyle Intervention for Diabetes Treatment: Five Year Outcomes from a Randomized Trial. J Clin Endocrinol Metab. 2020;105(3):866-876.

2. Schauer PR, Bhatt DL, Kashyap SR. Bariatric Surgery or Intensive Medical Therapy for Diabetes after 5 Years. N Engl J Med. 2017;376(7):641-651.

3. Sundbom M, Näslund E, Vidarsson B, et al. Low overall mortality during 10years of bariatric surgery: nationwide study on 63,469 procedures from the Scandinavian Obesity Registry. Surg Obes Relat Dis. 2020;16(1):6570 .

4. Wong JM, de Souza R, Kendall CW, et al. Colonic health: fermentation and short chain fatty acids. J Clin Gastroenterol. 2006;40(3):235-243.

5. American Medical Association (AMA). AMA Adopts New Policies on Second Day of Voting at Annual Meeting. USA: American Medical Association (AMA); 2013.

6. $\mathrm{Ng} \mathrm{M}$, Fleming $\mathrm{T}$, Robinson $\mathrm{M}$, et al. Global, regional, and national prevalence of overweight and obesity in children and adults during 1980 2013: a systematic analysis for the Global Burden of Disease Study 2013. Lancet. 2014;384(9945):766-781.

7. Stevens GA, Singh GM, Lu Y, et al. National, regional, and global trends in adult overweight and obesity prevalences. Popul Health Metr 2012;10(1):22.

8. Kelly T, Yang W, Chen CS, et al. Global burden of obesity in 2005 and projections to 2030. Int J Obes. 2008;32(9):1431-147.

9. Gregg EW, Chen H, Wagenknecht LE, et al. Association of an intensive lifestyle intervention with remission of type 2 diabetes. JAMA. 2012;308(23):2489-1496.

10. Knowler WC, Fowler SE, Hamman RF, et al. 10-year follow-up of diabetes incidence and weight loss in the Diabetes Prevention Program Outcomes Study. Lancet. 2009;374(9702):1677-1686.

11. Look AHEAD Research Group. Eight-year weight losses with an intensive lifestyle intervention: the look AHEAD study. Obesity (Silver Spring). 2014;22(1):5-13.
12. Yanovski SZ, Yanovski JA. Long-term drug treatment for obesity: a systematic and clinical review. JAMA. 2014;311(1):74-86.

13. de Freitas LM Jr, de Almeida EB Jr. Medicinal plants for the treatment of obesity: ethnopharmacological approach and chemical and biological studies. Am J Transl Res. 2017;9(5):2050-2064.

14. Balaji M, Ganjayi MS, Kumar GH, et al. A review on possible therapeutic targets to contain obesity: the role of phytochemicals. Obes Res Clin Pract. 2016;10(4):363-380.

15. Casacchia T, Scavello F, Rocca C, et al. Leopoldia comosa prevents metabolic disorders in rats with high-fat diet-induced obesity. Eur J Nutr. 2018;58(3):965-979.

16. Odunsi ST, Roque MV, Camilleri M, et al. Effect of alginate on satiation, appetite, gastric function and selected gut satiety hormones in overweight and obesity. Obesity. 2010;18(8):1579-1584.

17. Pérez-Jiménez A, Rufino-Palomares EE, Fernández-Gallego N, et al. Target molecules in 3T3-L1 adipocytes differentiation are regulated by maslinic acid, a natural triterpene from Olea europaea. Phytomedicine. 2016;23(12):1301-1311.

18. Roberfroid M, Gibson GR, Hoyles L, et al. Prebiotic effects: metabolic and health benefits. Br J Nutr. 2010;104(Suppl 2):S1-S63.

19. Slavin J. Fiber and prebiotics: mechanisms and health benefits. Nutrients. 2013;5(4):1417-1435.

20. Bianchi F, Larsen N, Tieghi TM, et al. In vitro modulation of human gut microbiota composition and metabolites by Bifidobacterium longum BB-46 and citric pectin. Food Res Int. 2019;120:595-602.

21. Cárdenas-Castro AP, Bianchi F, Tallarico-Adorno MA, et al. In vitro colonic fermentation of Mexican "taco" from corn-tortilla and black beans in a Simulator of Human Microbial Ecosystem (SHIME®) system. Food Res Int. 2019;118:81-88.

22. Kallus SJ, Brandt LJ. The intestinal microbiota and obesity. J Clin Gastroenterol. 2012;46(1):16-24.

23. Slavin JL. Dietary fiber and body weight. Nutrition. 2005;21(3):411-418.

24. Brusaferro A, Cozzali R, Orabona C, et al. Is It Time to Use Probiotics to Prevent or Treat Obesity? Nutrients. 2018;10(11):1613.

25. Zhou JC, Zhang XW. Akkermansia muciniphila: a promising target for the therapy of metabolic syndrome and related diseases. Chin J Nat Med. 2019;17(11):835-841. 